\title{
BMJ Open Association between same day discharge total knee and total hip arthroplasty and risks of cardiac/ pulmonary complications and readmission: a population-based observational study
}

\author{
Jiabin Liu (D) , ${ }^{1}$ Nabil Elkassabany, ${ }^{2}$ Jashvant Poeran, ${ }^{3}$ \\ Alejandro Gonzalez Della Valle, ${ }^{4}$ David H Kim, ${ }^{1}$ Daniel Maalouf, ${ }^{1}$ \\ Stavros Memtsoudis ${ }^{1}$
}

To cite: Liu J, Elkassabany N, Poeran J, et al. Association between same day discharge total knee and total hip arthroplasty and risks of cardiac/ pulmonary complications and readmission: a population-based observational study. BMJ Open 2019;9:e031260. doi:10.1136/ bmjopen-2019-031260

- Prepublication history for this paper is available online. To view these files, please visit the journal online (http://dx.doi org/10.1136/bmjopen-2019031260).

Received 24 April 2019 Revised 28 October 2019 Accepted 07 November 2019

D) Check for updates

(c) Author(s) (or their employer(s)) 2019. Re-use permitted under CC BY-NC. No commercial re-use. See rights and permissions. Published by BMJ.

For numbered affiliations see end of article.

Correspondence to Dr Jiabin Liu; liuji@hss.edu

\section{ABSTRACT}

Objective To determine if same-day discharge total knee arthroplasty (TKA) or total hip arthroplasty (THA) is not associated with increased risk of unplanned readmission and adverse outcomes within 30 days of surgery.

Design This is a population-based observational study. Setting Patients from 708 participating institutions who underwent primary TKA or primary THA between 2011 and 2017 were divided into three groups by length of stay (LOS 0,1 and $2-3$ days). All patients with LOS $>3$ days were excluded from the current study. Regression analysis and propensity score matching were performed.

Data sources American College of Surgeons-National Surgical Quality Improvement Programme database. Main outcomes and measures Primary outcomes included unplanned readmission and cardiac/pulmonary complications within 30 days of surgery.

Results We identified 226481 TKA (LOS $0=3118$, LOS $1=31404$, and LOS 2-3=191959) and 140557 THA patients ( $\operatorname{LOS} 0=2652$, LOS $1=29617$, and LOS 2-3=108288). There were no differences in 30-day mortality. After adjusting for relevant covariates, LOS 0 (compared with LOS 1) was associated with higher odds of cardiac/pulmonary complications in both TKA (OR 1.95, 95\% Cl 1.20 to 3.16; $0.67 \%$ vs $0.37 \%$ ) and THA (OR $1.96,95 \% \mathrm{Cl} 1.05$ to 3.64 ; $0.57 \%$ vs $0.26 \%$ ). There were no statistical differences in unplanned readmissions between LOS 0 and LOS 1 groups in TKA $(2.41 \%$ vs $2.31 \%)$ and THA (1.62\% vs $2.04 \%)$.

Conclusions LOS 0 discharge after TKA and THA was associated with higher odds of cardiac/pulmonary complications compared with LOS 1 discharge. While the overall burden of complications is relatively low, until future studies can confirm or challenge our findings, a measured approach is advisable when recommending discharge of patients on the same day of surgery.

\section{INTRODUCTION}

Joint arthroplasty is among the most commonly performed procedures in USA
Strength and limitations of this study

- Information on safety of same-day discharge after total knee arthroplasty (TKA) and total hip arthroplasty (THA) is lacking.

- This is the first comprehensive study to focus on length of stay (LOS) 0 and LOS 1 TKA and THA patients

- This is a population-based observational study, and cannot establish causal relationships.

with projections of continuous growth in parallel with an ageing population. Total knee arthroplasty (TKA) is projected at 3.48 million procedures annually, while total hip arthroplasty (THA) at 700000 per year by $2030 .{ }^{1}$ Until recently, TKA and THA were listed as Inpatient Only (IPO) procedures by the Centre for Medicare and Medicaid Service (CMS), which requires greater than 24 hours of postoperative care. Financial necessity, advances in surgical techniques, improved pain management and early physical rehabilitation have led to a continuous reduction in total hospital length of stay (LOS) after surgery. ${ }^{2}$ This trend has made ambulatory joint arthroplasty practice feasible. In fact, CMS removed TKA from the IPO list in January 2018 with the expectation of reducing healthcare cost. ${ }^{3}$ It is likely that CMS might remove THA from the IPO list in the near future, especially since the American Association of Orthopaedic Surgeons has also provided supportive statements for outpatient total hip arthroplasty. ${ }^{4}$ Such policy decisions by CMS cast significant pressure on 
hospitals and healthcare providers to fast track patients, and expedite a push towards performing surgery at freestanding ambulatory surgery centres.

However, practical and logistical concerns remain regarding the safety of fast track approaches, especially the true ambulatory practice with same day discharge. While mostly focused on patient selection and optimisation of peri-operative care guided under well-defined clinical pathways, actual outcome data are scarce. Several studies found no difference in short-term complications after comparing shorter inpatient stay with LOS $\geq 2$ days. ${ }^{5-7}$ Additional studies comparing admission status of outpatient versus inpatient, and concluded that outpatient joint arthroplasty is safe and effective. ${ }^{8-11}$ None of these studies focused on true ambulatory population (LOS 0 ) and the fast track group (LOS 1). One previous study by Otero et al included a small group of LOS 0 patients, and did not identify differences among TKA patients but increased complication rate in THA patients. ${ }^{12}$ Gromov et al studied 116 LOS 0 patients with matching cohort of 339 patients (LOS 1-9 days), and found no readmissions within 48 hours and comparable incidence of readmission within 90 days. ${ }^{13}$ However, these earlier studies were limited by the small sample size to be conclusive, and study cohort included emergent procedures, bilateral arthroplasties, or mixed TKA/THA patient population.

Given the current push towards same-say discharge after lower extremity joint arthroplasty surgery and lack of large-scale data on crucial comparisons, we therefore sought to study the safety of ambulatory surgical practice of TKA and THA with the access of several folds of more subjects to hopefully draw more convincing conclusion. For this purpose, we studied and compared complications and readmission rate and risk in patients discharged on the day of surgery (LOS 0 ) to those with a LOS of 1 day (LOS 1). We also included the standard practice group with LOS 2-3 days as a reference group. We hypothesised that there would be no difference in complications and readmission rates and risks among patients discharged same day of TKA or THA surgery.

\section{METHODS}

The population-based observational study follows the Strengthening the Reporting of Observational Studies in Epidemiology statement (second paragraph of the 'Patient and Public Involvement' section).

\section{Cohort description}

We acquired the data from the American College of Surgeons National Surgical Quality Improvement Program (NSQIP) from 2011 to 2017 (http://site.acsnsqip.org). NSQIP prospectively collects data on over 200 variables, including demographic information, comorbidities, intraoperative variables, 30-day postoperative complications and readmission. NSQIP conducted independent follow-ups of all registered patients for 30 days even after discharged from hospital, therefore NSQIP was able to capture postsurgical events for 30 days no matter whether patients were still in hospital or were discharged to other destination. NSQIP database does not include surgical procedures performed at ambulatory surgical centre as of 2017. To define our study cohort, we only included patients with the principal Current Procedural Terminology (CPT) code for primary TKA (CPT 27447) or primary THA (CPT 27130). We only included patients from 2011 to 2017, as the NSQIP dataset provides information on the readmission incidence within 30 days of surgery during this time frame.

There were a total of $n=232218$ and $n=141767$ entries for TKA and THA with LOS from 0 to 3 calendar days, respectively. We first excluded patients categorised as 'emergency' to establish a more homogeneous study cohort ( $\mathrm{n}=193$ and 435 respectively). We then excluded patients who received bilateral arthroplasty as defined by the relevant concurrent CPT code $(\mathrm{n}=5544$ and 775 respectively). The final cohort included 226481 and 140557 subjects for TKA and THA, respectively.

\section{Study variables}

Patients were separated into three groups based on LOS calculated based on calendar days (LOS 0 for same day discharge; LOS 1 for patients with next day discharge; and LOS 2-3 for patients with a traditional LOS of 2 to 3 days). The outcomes of interest were readmission within 30 days and six composite complication variables, including: wound infection, systemic infection, cardiac/pulmonary complications (including cardiac arrest requiring CPR, myocardial infarction, pulmonary embolism, unplanned intubation and/or on ventilator $>48$ hours), major complications (including any cardiac, pulmonary, central nerve system, renal, or systemic infection complications), any complication (including any complications enlisted in the NSQIP database), and any complication excluding blood transfusion.

\section{Statistical analysis}

Data analysis was executed using STATA V.14.2 statistical software (StataCorp LP, College Station, TX). Analysis of variance was used to analyse continuous variables.

Pearson $\chi^{2}$ tests were applied for categorical variables. After applying Bonferroni correction, $\mathrm{p}$ value less than $0.0036(0.05 / 14$ variables $)$ was used as the cut-off for statistical significance.

We next conducted single variable and multi-variable regression analysis to examine the impact of LOS on readmission and complications. The confounding variables included age, sex, race, body mass index (BMI), surgical duration, year of surgery and American Society of Anesthesiologists (ASA) classification. In the regression analysis we treated the LOS 1 group as the reference. The OR and $95 \%$ CI were reported. We elected to report output from the multi-variable regression analysis in the result section. To further evaluate robustness of our results, we also performed a propensity score matched analysis where the significant covariates were entered to calculate 
the propensity score to receive either same day (LOS 0) or fast track (LOS 1) surgery. We employed the Kernel matching algorithm based on the weighted average of all controls, and the weights are inversely proportional to the distance between the propensity scores.

\section{Patient and public involvement}

The current study involved prospectively collected patient information without any identifiable patient specific information. None of these included study subjects would benefit from the current study. However, future patients may benefit from the knowledge highlighted in the current study once it is publicly available. Patients were not involved in the design and conduct of current study. All patient related information was de-identified from the database to preserve privacy.

\section{RESULTS}

We identified $\mathrm{n}=226481$ primary TKA (LOS $0=3118$, LOS $1=31404$, and LOS 2-3=191959) and 140557 primary THA patients (LOS $0=2652$, LOS $1=29617$, and LOS $2-3=108288$ ), respectively. There were no major clinically significant differences in the comorbidity burden between LOS 0 and LOS 1 groups, while LOS 2-3 group carried a higher comorbidity burden (table 1 ). Between 2011 and 2017, LOS trended downwards, with an increasing number of patients being discharged on the day of surgery or the next day (TKA $1.04 \%$ in 2011, and $26.55 \%$ in 2017 ; THA $3.44 \%$ in 2011 , and $34.91 \%$ in 2017, respectively). The discharge destination was most frequently to home among the various TKA groups (LOS 0 group $89.48 \%$, LOS 1 group $97.62 \%$ and $76.39 \%$ in LOS 2-3 group). Home discharge was the most prominent disposition in THA as well (LOS 0 group 94.72\%, LOS 1 group $97.92 \%$ and $78.37 \%$ in LOS 2-3 group). There were no differences in 30-day mortality in either TKA (table 2) or THA groups (table 3). The incidences of 30-day major complications and unplanned readmissions were low in the LOS 1 discharge group $(0.53 \%$ and $2.31 \%$ in TKA; $0.43 \%$ and $2.04 \%$ in THA respectively). There were no statistically significant differences in unplanned readmission between LOS 0 and LOS 1 group in TKA patients $(2.41 \%$ vs $2.31 \%$ in TKA, OR $1.10,95 \%$ CI 0.86 to 1.42 , table 2$)$, nor among THA patients (1.62\% vs $2.04 \%$ in THA, OR $0.84,95 \%$ CI 0.60 to 1.16 , table 3 ). LOS 2-3 group otherwise carried the highest incidence of unplanned readmission (tables 2 and 3 ).

Tables 2 and 3 also provide results from the univariable regression, multivariable regression and the propensity score matching analysis comparing the LOS 0 to the LOS 1 groups. The LOS 0 group was associated with higher odds of cardiac/pulmonary complications in both TKA (OR 1.95, 95\% CI 1.20 3.16; unadjusted prevalence 0.67\% vs $0.37 \%$, table 2) and THA (OR $1.96,95 \%$ CI 1.05 to 3.64; unadjusted prevalence $0.57 \%$ vs $0.26 \%$, table 3 ) when compared with the LOS 1 group. Propensity score matching analysis confirmed such increased incidence of cardiac/pulmonary complications in LOS 0 group (tables 2 and 3). The incidence was even higher when compared with the LOS 2-3 group (tables 2 and 3).

LOS 0 group was associated with higher odds of major complications in TKA recipients (OR 1.94, 95\% CI 1.29 to 2.92, table 2), but not in THA patients (OR 1.55, 95\% CI 0.90 to 2.67, table 3) compared with LOS 1 patients. Similar patterns of differences were also observed in the outcomes for any complications, and any complications excluding transfusion. These differences were statistically significant only in TKA (table 2) but not in THA (table 3). Propensity score matching analysis further confirmed all significances (tables 2 and 3). There were no differences in wound infection and systemic infection between LOS 0 and LOS 1 groups in either TKA or THA.

\section{DISCUSSION}

In this analysis of data collected by NSQIP, we present data using population data that challenge the assumed safety of same day discharge after TKA or THA surgery. Our analysis showed somewhat surprising results that LOS 0 group had higher risks of cardiac/pulmonary complications within 30 days after surgery in both TKA and THA, compared with patients in the LOS 1 group. Our study also identified significantly increased odds for major complications with LOS 0 discharge status compared with LOS 1 group among TKA recipients.

The average LOS after TKA decreased from 3.42 days in 2011 to 2.38 days in 2017, and from 3.54 days in 2011 to 2.31 days in 2017 after THA surgery (NSQIP data). Accordingly, more patients received fast-track care in more recent years. Previous studies have not shown a difference in readmission rates and complications among fast-track TKA and THA patients. ${ }^{14-19}$ Definition of fasttrack practice has also been evolving, from previously LOS 2-3 days to as short as LOS 1 day. It is foreseeable that fast-track practice will gear towards LOS 0 day status in the future. Otero et al studied patients from 2011 and 2013, which they concluded that there were no differences in readmission and 30-day complication in TKA between LOS 0 and LOS 1 status, while THA patients with LOS 0 status were associated with a higher 30-day complication rate. ${ }^{12}$ However, this study is limited by the small number of patients in the LOS 0 and LOS 1 groups. In addition, the authors did not exclude patients with emergent admission status and patients who received bilateral arthroplasty procedures. Lately, Gromov et alstudied LOS 0 patients with a matching cohort of controls with LOS 1-9 days. ${ }^{13}$ The authors concluded that readmission rates were comparable. However, such comparison might not be fair since LOS 1-9 patients, especially patients with longer LOS usually have indications for hospitalisation. The staying in hospital would decrease chances of readmission, nor with recorded diagnosis for readmission. Other researchers have attempted to study the difference in complications in arthroplasty based on the admission status either as outpatient or inpatient. ${ }^{20}$ However, such 


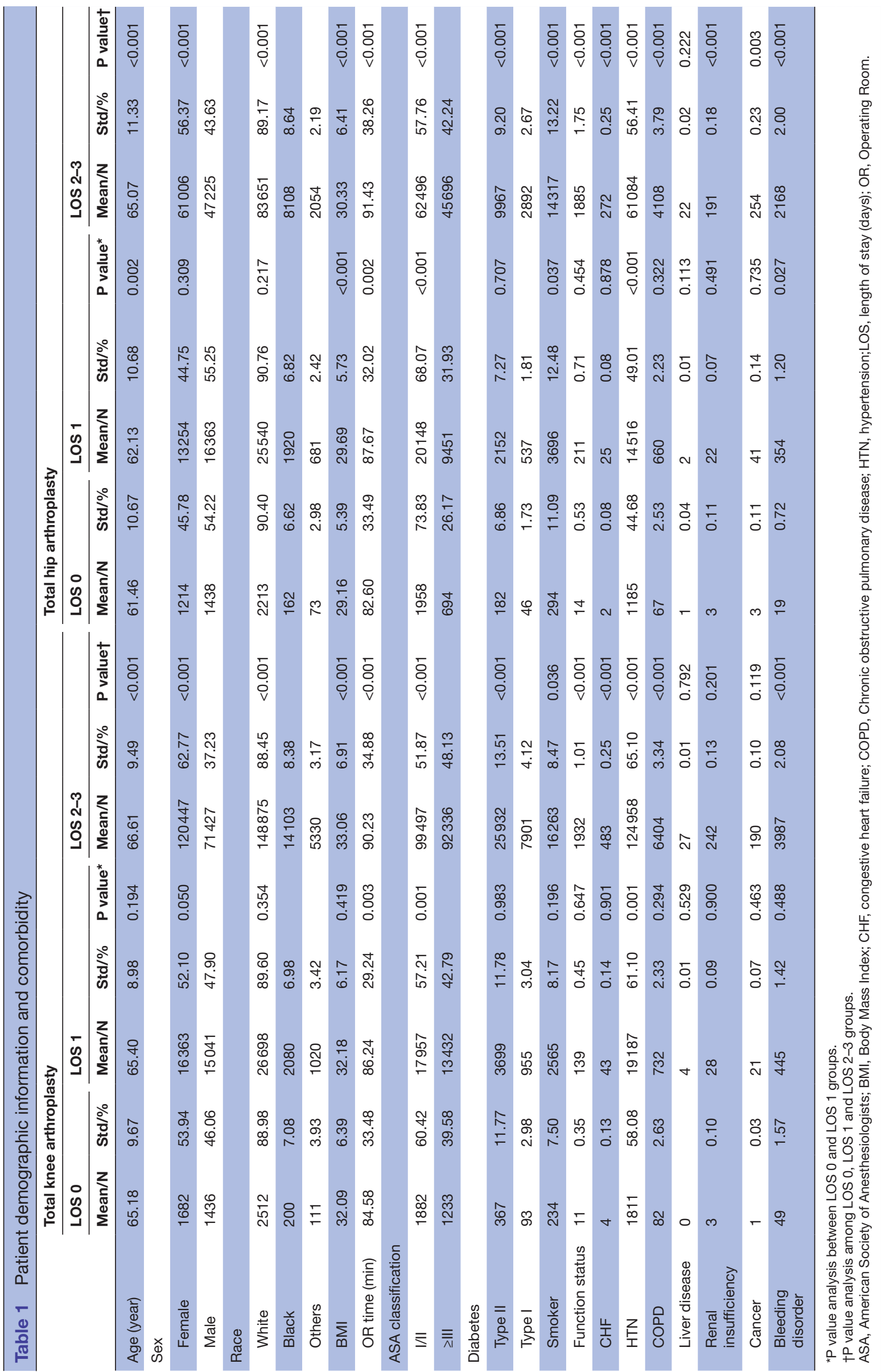




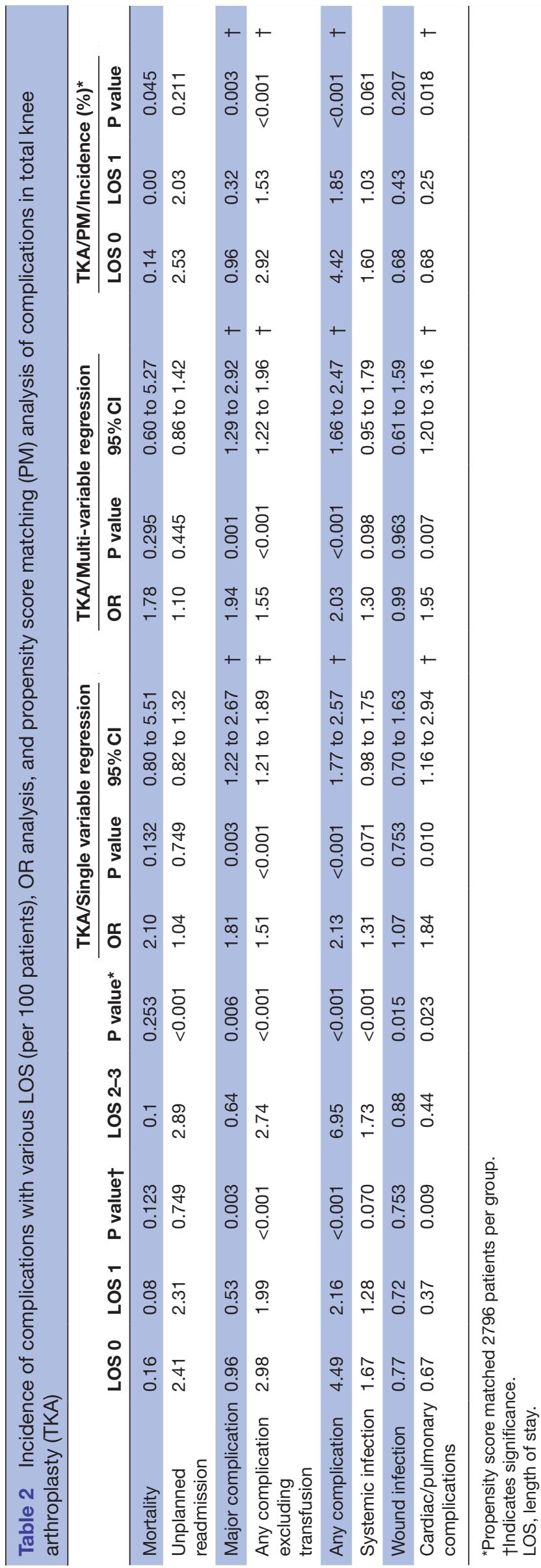

categorisation among arthroplasty recipients was arbitrary which was most likely influenced by the type of patients' insurance. Nonetheless, concerns remained among clinicians regarding the balance of safe clinical practice and fast-track efficiency.

In order to achieve these goals, clinicians have attempted to identify patients at risk of readmission or complications, and thus triage them accordingly. Many independent risk factors have been identified, including advanced age, gender, high BMI, increased ASA classification, the presence of chronic obstructive pulmonary disease, congestive heart failure, coronary artery disease, cirrhosis and chronic kidney disease. $.^{512} 21-25$ In addition, poor living conditions, use of mobility aids and social economic factors are also likely to influence LOS and outcomes. ${ }^{622} 26$ Clinicians further developed prediction models to determine a patient's candidacy for fasttrack surgical care with moderate success. ${ }^{5627}$ It should be mentioned, however, that some data suggest that the majority of patients suffering from a complication after joint arthroplasty may not have any identifiable risk factors, ${ }^{21}$ thus putting strategies currently being used to identify patients at risk into question.

The majority of major complications, such as cardiac/ pulmonary complications, likely occur past 24 hours, and more likely peak on postoperative day 2 to $3 .^{528} 29$ This timeframe may therefore fall outside the in-hospital observation period as it relates to fast-track patients. It also has been shown that over $50 \%$ of patients with major complications do not carry any of these predisposing risk factors. ${ }^{21}$ Therefore, identifying risk factors and risk stratification of patient populations may be of limited use in predicting successful fast-track patients without risk of readmission or complications. The current approach seeks to identify higher risk patients and subsequently exclude them from the fast track pathway. This is supported by our findings that patients in the LOS2-3 group have higher comorbidity burden and are older than those in the LOS 0 and LOS 1 groups.

Many institutions have established enhanced recovery after surgery pathways for TKA and THA. These pathways seek to improve outcomes by standardising the routine use of potentially beneficial interventions that improve outcomes and by employing patient selection strategies. Consequently they include younger and healthier patients with sufficient social support to facilitate early discharge. However, such approaches may not be sufficient to reconcile them with unaltered or lower level of complications while gearing towards true ambulatory surgical model.

We conducted this NSQIP data analysis with the hypothesis that ambulatory patients were not at increased risk comparing to other fast track surgical patients after TKA or THA. It is reasonable to assume that these fasttracked patients were carefully selected without major comorbidity concerns. Further, it is reasonable to assume that these patients met the discharge criteria established across various institutions. Assuming such safe practice model were established and applied, our findings raise 


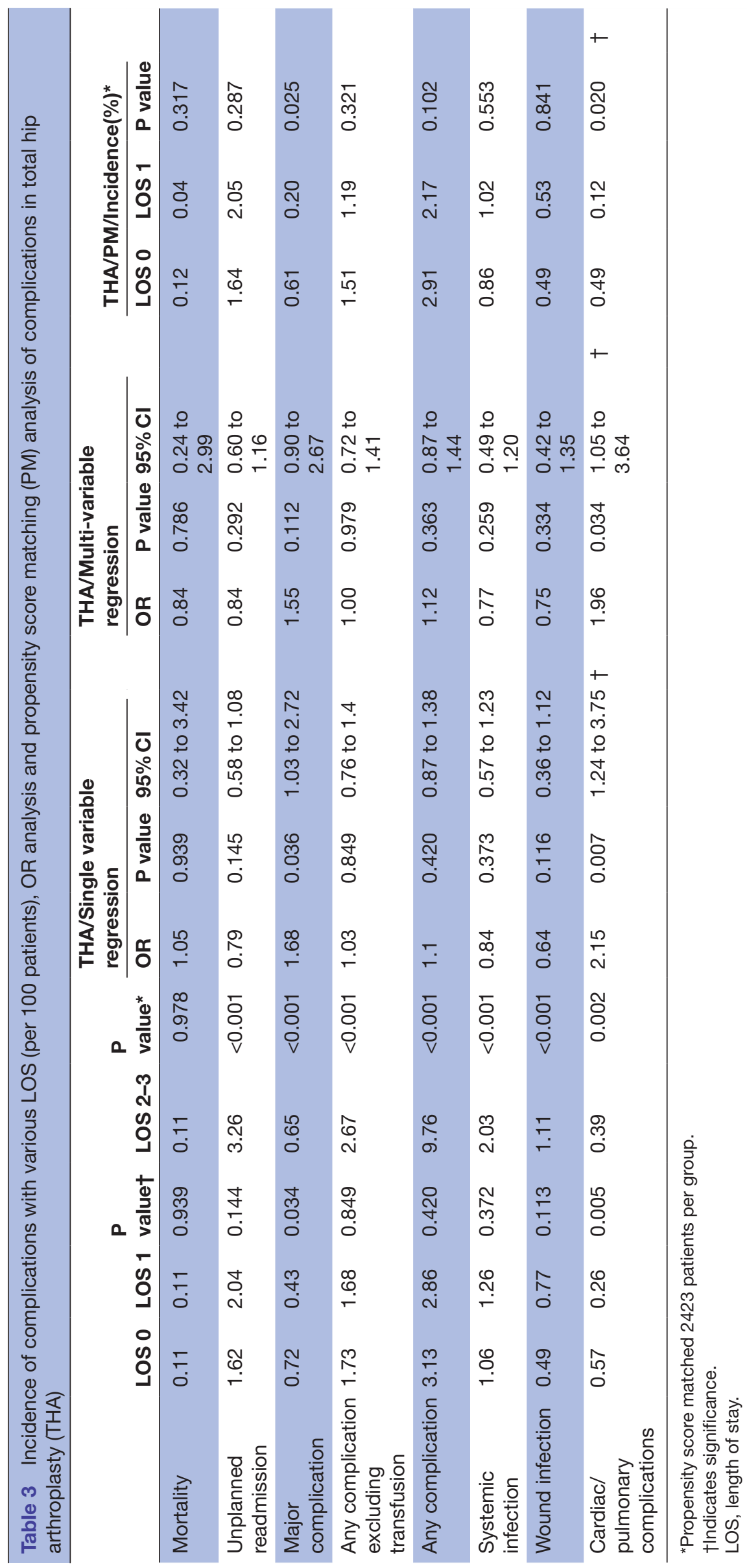


concern regarding the safety of same day discharge after TKA or THA surgery. This is especially problematic if the increased risk of complications among this group is related to gaps in continuity of care and lack of necessary early intervention when indicated. However, despite this possibility, our data is not able to establish this causal relationship at this time, and future studies are needed to identify the aetiology and mechanism by which complications may develop.

Our study has several limitations. First, this is a retrospective cohort study and related limitations in respect to establishing causality apply. Although NSQIP has rigorous quality measures to ensure high quality data collection, there was still missing information on several interesting pre-existing comorbidity variables, such as stroke and myocardial infarction. Our study is therefore limited by the accuracy and completeness of data collection, inclusion of available variables and recorded information only. Second, patients were categorised retrospectively based on their actual LOS determined by calendar days. Future research is indicated to prospectively assign clinical pathways and compare readmission and complications. Third, NSQIP only contains outcome information within 30 days, thus outcomes beyond this point but still related to the index procedure remain elusive. Fourth, NSQIP prohibits identifying hospital and surgeon, while studying surgical volume, inpatient hospital versus free-standing surgical centre, and other practice pattern might be insightful. Last, readmission indicator in NSQIP database only included inpatient readmission. Information on emergency department visit would also be important. However, it is beyond the scope of our analysis.

\section{CONCLUSION}

Our study is the first comprehensive study to focus on LOS 0 TKA and THA patients. Although same day discharge after TKA and THA surgery is not associated with increased risk of unplanned readmission, these patients carry increased risk of complications. Therefore, the current trend towards increasing discharges on the same day of surgery after TKA and THA should be approached with caution and requires reconsideration. Future prospective studies are needed to confirm our finding and identify if ambulatory joint arthroplasty is associated with acceptable risk for complications and readmissions, as well as its financial impact on our healthcare system.

\section{Author affiliations}

${ }^{1}$ Department of Anesthesiology, Critical Care \& Pain Management, Hospital for Special Surgery, Weill Medical College of Cornell University, New York City, New York, USA

${ }^{2}$ Department of anesthesiology and critical care, University of Pennsylvania, Philadelphia, Pennsylvania, USA

${ }^{3}$ Institute for Healthcare Delivery Science, Department of Population Health Science and Policy, Icahn School of Medicine at Mount Sinai, New York City, New York, USA ${ }^{4}$ Department of orthopedic surgery, Hospital for Special Surgery, New York City, New York, USA

\section{Twitter Jiabin Liu @JbLiuJb}

Acknowledgements The authors would like to thank Haoyan Zhong, BS, MPH, for her expert support in statistical issues. The authors also would like to thank Dr. Christopher L. Wu for his insightful review of the manuscript.

Contributors $\mathrm{JL}$ and NE were involved in attaining data from NSQIP. JL, NE, AGDV and SM were involved in designing the study. JL analysed data with help from JP and SM. All authors contributed to the interpretation of the results, including $\mathrm{JL}$, $\mathrm{NE}, \mathrm{JP}, \mathrm{AGDV}$, DHK, DM and SGM. All authors reviewed, revised and approved the final document. JL and SM are the study guarantors, and take responsibility for the completeness of the data and the accuracy of the analysis.

Funding The authors have not declared a specific grant for this research from any funding agency in the public, commercial or not-for-profit sectors.

Competing interests None declared.

Patient consent for publication Not required.

Ethics approval The study was exempted by the institutional review board (IRB\# 2017-0716) as data accessed and analysed were de-identified. Results will be communicated through publication in scientific journal and conference.

Provenance and peer review Not commissioned; externally peer reviewed.

Data availability statement Data may be obtained from a third party and are not publicly available.

Open access This is an open access article distributed in accordance with the Creative Commons Attribution Non Commercial (CC BY-NC 4.0) license, which permits others to distribute, remix, adapt, build upon this work non-commercially, and license their derivative works on different terms, provided the original work is properly cited, appropriate credit is given, any changes made indicated, and the use is non-commercial. See: http://creativecommons.org/licenses/by-nc/4.0/.

ORCID iD

Jiabin Liu http://orcid.org/0000-0002-1029-2786

\section{REFERENCES}

1 Kurtz S, Ong K, Lau E, et al. Projections of primary and revision hip and knee arthroplasty in the United States from 2005 to 2030. J Bone Joint Surg Am 2007;89:780-5.

2 Sloan M, Sheth NP. Length of stay and inpatient mortality trends in primary and revision total joint arthroplasty in the United States, 2000-2014. J Orthop 2018;15:645-9.

3 CMS. January 2018 update of the hospital outpatient prospective payment system (OPPS) 2017.

4 AAOS. Medicare program; Hospital outpatient prospective payment and ambulatory surgical center payment systems and quality reporting program; 2017.

5 Courtney PM, Rozell JC, Melnic CM, et al. Who should not undergo short stay hip and knee arthroplasty? risk factors associated with major medical complications following primary total joint arthroplasty. $J$ Arthroplasty 2015;30:1-4.

6 Gronbeck CJ, Cote MP, Halawi MJ. Predicting inpatient status after total hip arthroplasty in Medicare-Aged patients. J Arthroplasty 2019;34:249-54.

7 Duchman KR, Gao Y, Pugely AJ, et al. Differences in shortterm complications between unicompartmental and total knee arthroplasty: a propensity score matched analysis. J Bone Joint Surg Am 2014;96:1387-94.

8 Lovecchio F, Alvi H, Sahota S, et al. Is outpatient arthroplasty as safe as fast-track inpatient arthroplasty? A propensity score matched analysis. J Arthroplasty 2016;31:197-201.

9 Hoffmann JD, Kusnezov NA, Dunn JC, et al. The shift to same-day outpatient joint arthroplasty: a systematic review. J Arthroplasty 2018;33:1265-74.

10 Courtney PM, Froimson MI, Meneghini RM, et al. Can total knee arthroplasty be performed safely as an outpatient in the Medicare population? J Arthroplasty 2018;33:S28-31.

11 Courtney PM, Boniello AJ, Berger RA. Complications following outpatient total joint arthroplasty: an analysis of a national database. J Arthroplasty 2017;32:1426-30.

12 Otero JE, Gholson JJ, Pugely AJ, et al. Length of hospitalization after joint arthroplasty: does early discharge affect complications and readmission rates? J Arthroplasty 2016;31:2714-25.

13 Gromov K, Jørgensen CC, Petersen PB, et al. Complications and readmissions following outpatient total hip and knee arthroplasty: 
a prospective 2-center study with matched controls. Acta Orthop 2019;90:281-5.

14 Kehlet H, Wilmore DW. Evidence-Based surgical care and the evolution of fast-track surgery. Ann Surg 2008;248:189-98.

15 Klingenstein GG, Schoifet SD, Jain RK, et al. Rapid discharge to home after total knee arthroplasty is safe in eligible Medicare patients. J Arthroplasty 2017;32:3308-13.

16 Glassou EN, Pedersen AB, Hansen TB. Risk of re-admission, reoperation, and mortality within 90 days of total hip and knee arthroplasty in fast-track departments in Denmark from 2005 to 2011. Acta Orthop 2014;85:493-500.

17 Kiskaddon EM, Lee JH, Meeks BD, et al. Hospital discharge within 1 day after total joint arthroplasty from a Veterans Affairs Hospital does not increase complication and readmission rates. J Arthroplasty 2018;33:1337-42.

18 Sutton JC, Antoniou J, Epure LM, et al. Hospital discharge within 2 days following total hip or knee arthroplasty does not increase Major-Complication and readmission rates. J Bone Joint Surg Am 2016;98:1419-28.

19 Yang G, Chen W, Chen W, et al. Feasibility and safety of 2day discharge after fast-track total hip arthroplasty: a Chinese experience. J Arthroplasty 2016;31:1686-92.

20 Arshi A, Leong NL, D'Oro A, et al. Outpatient total knee arthroplasty is associated with higher risk of perioperative complications. $J$ Bone Joint Surg Am 2017;99:1978-86.

21 Parvizi J, Mui A, Purtill JJ, et al. Total joint arthroplasty: when do fatal or near-fatal complications occur? J Bone Joint Surg Am 2007;89:27-32.
22 Jørgensen CC, Kehlet H, Lundbeck Foundation Centre for Fast-track Hip and Knee Replacement Collaborative Group. Role of patient characteristics for fast-track hip and knee arthroplasty. Br J Anaesth 2013;110:972-80.

23 Rozell JC, Courtney PM, Dattilo JR, et al. Late complications following elective primary total hip and knee arthroplasty: who, when, and how? J Arthroplasty 2017;32:719-23.

24 Lovald ST, Ong KL, Lau EC, et al. Patient selection in short stay total hip arthroplasty for Medicare patients. J Arthroplasty 2015;30:2086-91.

25 Sikora-Klak J, Gupta A, Bergum C, et al. The evaluation of comorbidities relative to length of stay for total joint arthroplasty patients. J Arthroplasty 2017;32:1085-8.

26 Inneh IA, lorio R, Slover JD, et al. Role of sociodemographic, comorbid and intraoperative factors in length of stay following primary total hip arthroplasty. J Arthroplasty 2015;30:2092-7.

27 Schilling PL, Bozic KJ. Development and validation of perioperative risk-adjustment models for hip fracture repair, total hip arthroplasty, and total knee arthroplasty. J Bone Joint Surg Am 2016;98:e2.

28 Jørgensen CC, Kehlet H, Lundbeck Foundation Centre for Fasttrack Hip and Knee replacement collaborative group. Early thromboembolic events $\leq 1$ week after fast-track total hip and knee arthroplasty. Thromb Res 2016;138:37-42.

29 Petersen PB, Kehlet H, Jørgensen CC, et al. Myocardial infarction following fast-track total hip and knee arthroplasty-incidence, time course, and risk factors: a prospective cohort study of 24,862 procedures. Acta Orthop 2018;89:603-9. 